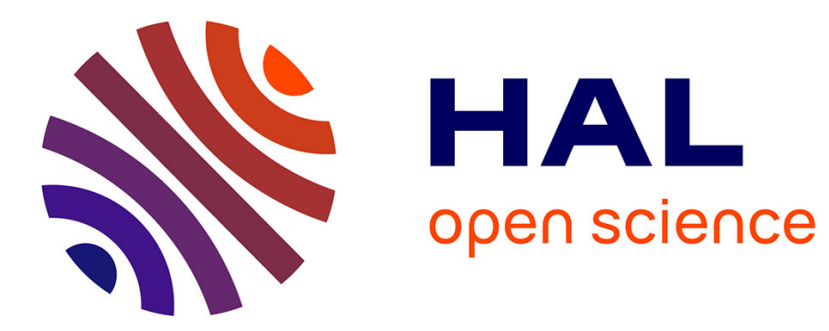

\title{
On quasi-elastic scattering of slow neutrons in molecular liquids
}

\author{
M. Utsuro
}

\section{To cite this version:}

M. Utsuro. On quasi-elastic scattering of slow neutrons in molecular liquids. Journal de Physique Lettres, 1977, 38 (15), pp.317-320. 10.1051/jphyslet:019770038015031700 . jpa-00231385

\section{HAL Id: jpa-00231385 https://hal.science/jpa-00231385}

Submitted on 1 Jan 1977

HAL is a multi-disciplinary open access archive for the deposit and dissemination of scientific research documents, whether they are published or not. The documents may come from teaching and research institutions in France or abroad, or from public or private research centers.
L'archive ouverte pluridisciplinaire HAL, est destinée au dépôt et à la diffusion de documents scientifiques de niveau recherche, publiés ou non, émanant des établissements d'enseignement et de recherche français ou étrangers, des laboratoires publics ou privés. 


\title{
ON QUASI-ELASTIC SCATTERING OF SLOW NEUTRONS IN MOLECULAR LIQUIDS
}

\author{
M. UTSURO \\ Research Reactor Institute, Kyoto University, Kumatori-cho, Sennan-gun, Osaka, 590-04 Japan
}

(Reçu le 21 février 1977, révisé le 12 mai 1977, accepté le 1er juillet 1977)

\begin{abstract}
Résumé. - Des spectres de diffusion quasi élastique de neutrons avec élargissement par rotation moléculaire dans le liquide sont étudiés dans le cadre du "step » formalisme et de l'approximation de la diffusion rotationnelle. Ils sont décomposés en distributions Lorentziennes en utilisant des relations simples. Les résultats du calcul concernant les largeurs en énergie et les intensités permettent d'envisager l'observation séparée de ces termes dans le cas du benzène liquide.
\end{abstract}

\begin{abstract}
The rotational broadened quasi-elastic scattering spectrum of neutrons in a molecular liquid which is studied in the framework of the step formalism and the rotational diffusion approximation can be decomposed into simple Lorentzian distributions. The possibility of separately observing these terms in the experiment is discussed with respect to the numerical results of their energy broadening and amplitude for the case of liquid benzene.
\end{abstract}

1. Introduction. - Quasi-elastic scattering of slow neutrons relating to long time behaviour in liquid dynamics gives information concerning diffusive motions or possible collisional motions in the region of about a few pico-seconds or more in the liquid. In the case of molecular liquids such as liquid hydrocarbons, rotational motions of molecules can also affect the energy broadening or the intensity of the quasi-elastic scattering, and many papers have already been devoted to this problem [1-6]. Hydrogenous molecular liquids were mostly considered and incoherent quasi-elastic scattering with the assumption of independence between translational and rotational motions of the molecules has been studied. In this case, the scattering law for a quasi-elastic process $S_{\mathrm{q} \cdot \mathrm{cl}}(\kappa, \omega)$ with momentum transfer $\kappa$ and energy transfer $\hbar \omega$ can be written [6]

$$
\begin{aligned}
& S_{\mathrm{q} . \mathrm{el}}(\kappa, \omega)=F(\kappa) \frac{1}{\pi} \frac{D \kappa^{2}}{\omega^{2}+\left(D \kappa^{2}\right)^{2}}+ \\
& +\left(\sum_{n}^{\text {terms }} \begin{array}{c}
\text { broadened } \\
\text { terms }
\end{array}\right) \otimes \frac{1}{\pi} \frac{D \kappa^{2}}{\omega^{2}+\left(D \kappa^{2}\right)^{2}},
\end{aligned}
$$

where a simple diffusion approximation was used for the translational motions of the molecules and $D$ is the self-diffusion coefficient in the liquid. $\otimes$ denotes convolution concerning the frequency $\omega$.

In this letter, one of the possible methods of studying the detailed structure of the second term in eq. (1) is presented by using a step formalism [7,3] and the rotational diffusion approximation [1] for the description of the rotational motions in the liquid.

2. Lorentzian decomposition of rotational broadened spectra. - For the analysis of the contributions of the rotational motions to the quasi-elastic scattering spectra, the step formalism of Singwi-Sjölander [7] and Larsson [3] is employed. The fundamental lines of the step formalism applied to molecular rotations have already been studied by Larsson [3], and therefore, the practical result with the assumptions of translational and also rotational diffusions for molecular motions in liquid will be derived here quite briefly.

The orientational distribution function $g\left(\Omega_{0}, \Omega, t\right)$ and $h\left(\Omega_{0}, \Omega, t\right)$ for a nucleus around the molecular center of mass during the periods of librational motions with an average lifetime $\tau_{0}$ and reorientational motions with that of $\tau_{1}$, respectively, can be written as follows as spherical harmonics expansions $[1,3]$ :

$$
\begin{aligned}
& g\left(\Omega_{0}, \Omega, t\right)=\sum_{l=0}^{\infty} F_{l}^{\mathrm{lib}} Y_{l m}(\Omega) Y_{l m}^{*}\left(\Omega_{0}\right) . \\
& h\left(\Omega_{0} . \Omega, t\right)=\sum_{l=0}^{\infty} F_{l}^{\mathrm{reo}} Y_{l m}(\Omega) Y_{l m}^{*}\left(\Omega_{0}\right) \cdot \exp \left[-l(l+1) D_{\mathrm{r}} t\right],
\end{aligned}
$$


where $D_{\mathrm{r}}$ is the rotational diffusion coefficient. Following Larsson [3] and with the present distribution functions of eq. (2) the result of quasi-elastic scattering law is :

$$
\begin{aligned}
& \pi S_{\mathrm{q} . \mathrm{el}}(\kappa, \omega)=j_{0}^{2}(\kappa b) \frac{D \kappa^{2}}{\omega^{2}+\left(D \kappa^{2}\right)^{2}}+\frac{1}{\tau_{0}+\tau_{1}} \sum_{l=1}^{\infty}(2 l+1) j_{l}^{2}(\kappa b) \times \\
& \times \operatorname{Re}\left[\frac{\frac{F_{l}^{\mathrm{lib}} \tau_{0}^{2}}{1+D \kappa^{2} \tau_{0}+i \omega \tau_{0}}+\frac{F_{l}^{\mathrm{reo}} \tau_{1}^{2}}{1+\left[D \kappa^{2}+l(l+1) D_{\mathrm{r}}\right] \tau_{1}+i \omega \tau_{1}}+\frac{2 F_{l}^{\mathrm{lib}} F_{l}^{\mathrm{reo}} \tau_{0} \tau_{1}}{\left\{1+D \kappa^{2} \tau_{0}+i \omega \tau_{0}\right\}\left\{1+\left[D \kappa^{2}+l(l+1) D_{\mathrm{r}}\right] \tau_{1}+i \omega \tau_{1}\right\}}}{1-\frac{F_{l}^{\mathrm{lib}}}{1+D \kappa^{2} \tau_{0}+i \omega \tau_{0}} \cdot \frac{F_{l}^{\text {reo }}}{1+\left[D \kappa^{2}+l(l+1) D_{\mathrm{r}}\right] \tau_{1}+i \omega \tau_{1}}}\right]
\end{aligned}
$$

where $b$ is the distance between the scattering nucleus and the molecular centre of mass.

Now, the complicated form of the scattering law eq. (3) can be decomposed into a much simpler form of a summation of Lorentzian distributions

$$
\begin{aligned}
& \pi S_{\mathrm{q} . \mathrm{el}}(\kappa, \omega)=j_{0}^{2}(\kappa b) \frac{D \kappa^{2}}{\omega^{2}+\left(D \kappa^{2}\right)^{2}}+ \\
& \quad+\sum_{l=1}^{\infty}(2 l+1) j_{l}^{2}(\kappa b)\left[\frac{\alpha_{l} \cdot\left(\Delta \omega_{l a}+D \kappa^{2}\right)}{\omega^{2}+\left(\Delta \omega_{l a}+D \kappa^{2}\right)^{2}}+\frac{\beta_{l} \cdot\left(\Delta \omega_{l b}+D \kappa^{2}\right)}{\omega^{2}+\left(\Delta \omega_{l b}+D \kappa^{2}\right)^{2}}\right]
\end{aligned}
$$

where

$$
\begin{aligned}
\Delta \omega_{l a, l b} & =\frac{1}{2}\left\{\frac{1}{\tau_{0}}+\frac{1}{\tau_{1}}+l(l+1) D_{\mathrm{r}} \pm \sqrt{\left.\left[\frac{1}{\tau_{0}}-\frac{1}{\tau_{1}}-l(l+1) D_{\mathrm{r}}\right]^{2}+\frac{4 F_{l}^{\mathrm{lib}} F_{l}^{\mathrm{reo}}}{\tau_{0} \tau_{1}}\right\}}\right. \\
\left.\begin{array}{c}
\alpha_{l} \\
\beta_{l}
\end{array}\right\} & =\frac{1}{2\left(\tau_{0}+\tau_{1}\right)} \quad\left\{F_{l}^{\mathrm{lib}} \tau_{0}+F_{l}^{\mathrm{reo}} \tau_{1} \pm \frac{4 F_{l}^{\mathrm{lib}} F_{l}^{\mathrm{reo}}+\left(F_{l}^{\mathrm{lib}} \tau_{1}-F_{l}^{\mathrm{reo}} \tau_{0}\right)\left[\frac{1}{\tau_{0}}-\frac{1}{\tau_{1}}-l(l+1) D_{\mathrm{r}}\right]}{\sqrt{\left[\frac{1}{\tau_{0}}-\frac{1}{\tau_{1}}-l(l+1) D_{\mathrm{r}}\right]^{2}+\frac{4 F_{l}^{\mathrm{lib}} F_{l}^{\mathrm{reo}}}{\tau_{0} \tau_{1}}}}\right\} .
\end{aligned}
$$

In the present case, eq. (4) means that the quasielastic broadenings of all the terms including the rotational contributions show the same $\kappa^{2}$-dependence, when decomposed into Lorentzians. That is, each higher order term has an energy broadening which is increased over the fundamental term $2 \hbar D \kappa^{2}$ by a constant value. Furthermore, eq. (4) means that the coefficients $\alpha_{l}$ and $\beta_{l}$ do not vary with changing $\kappa^{2}$.

3. Some physical considerations. - The present result of the Lorentzian decomposition comes from the expansion of the orientational distribution functions into series of orthonormal spherical harmonics and also from the exponential time-dependences of eq. (2) and of the decay functions for each of the steps. Because of the orthogonality in the expansion, the time-dependences in the distribution functions are coupled only between the terms of the same order of $l$, and further the exponential property leads to linear coupling between these time-dependences. Since two types of rotational motions, say librational and reorientational motions, are considered here, the result gives two characteristic time-constants for each $l$-th mode, which appear in the scattering law of eq. (4) as two kinds of energy broadenings for each $l$.
The above mentioned consideration also defines the limit of applicability of the present result. Since the behaviours in the short time region in each of the steps are not described correctly by eq. (2), the result can not give the exact value in the large transferred-energy region related to possible inelastic processes of librational or vibrational motions. Possible free-rotational behaviour in the short time region for the reorientational motions is also not taken into account in eq. (2). Concerning these points, one can easily test eq. (4) for 0 -th order sum rule, since

$\alpha_{l}+\beta_{l}=\frac{F_{l}^{\mathrm{lib}} \tau_{0}+F_{l}^{\mathrm{reo}} \tau_{1}}{\tau_{0}+\tau_{1}} \leqslant 1,\left(F_{l}^{\mathrm{lib}}, F_{l}^{\mathrm{reo}} \leqslant 1\right)$

This difference of the value for $\alpha_{l}+\beta_{l}$ from unity shows one of the defects of the present scattering law in large transferred-energy region. Furthermore, it is well known that the Lorentzian distribution can not satisfy higher order sum rules. In this way, the present result should not be applied in the region of too large a transferred-energy, say in the region of possible inelastic scattering due to librational or vibrational motions. 
If these precautions are well considered, the present simple expression for the structure of quasi-elastic scattering spectrum obtained from step formalism and diffusion approximations suggests an important point concerning the analysis of the experimental results for molecular liquids. If the experimental results of quasielastic scattering with sufficiently high accuracy and fine resolution can be decomposed into Lorentzian terms with energy broadenings of constant differences for changes of $\kappa^{2}$, then the long-time properties of molecular motions in the liquid should be very near to those described in the present approach. If, on the other hand, the result consists of a sharper component corresponding to the first term in eq. (1) and a remaining component of non-Lorentzian form or of a more complicated energy broadening than that in eq. (4), then several possibilities, such as molecular rotations including large angle free rotations, strong anisotropy in orientational distributions, or non-exponential decay of each of the steps, should be considered in the liquid. Of course, observation of mixtures of various higher order terms due to insufficient experimental resolution will also lead to similar complicated results.

4. Numerical examples. - In order to study the problem in more detail, typical values for the various parameters in eq. (4) are selected referring to the case

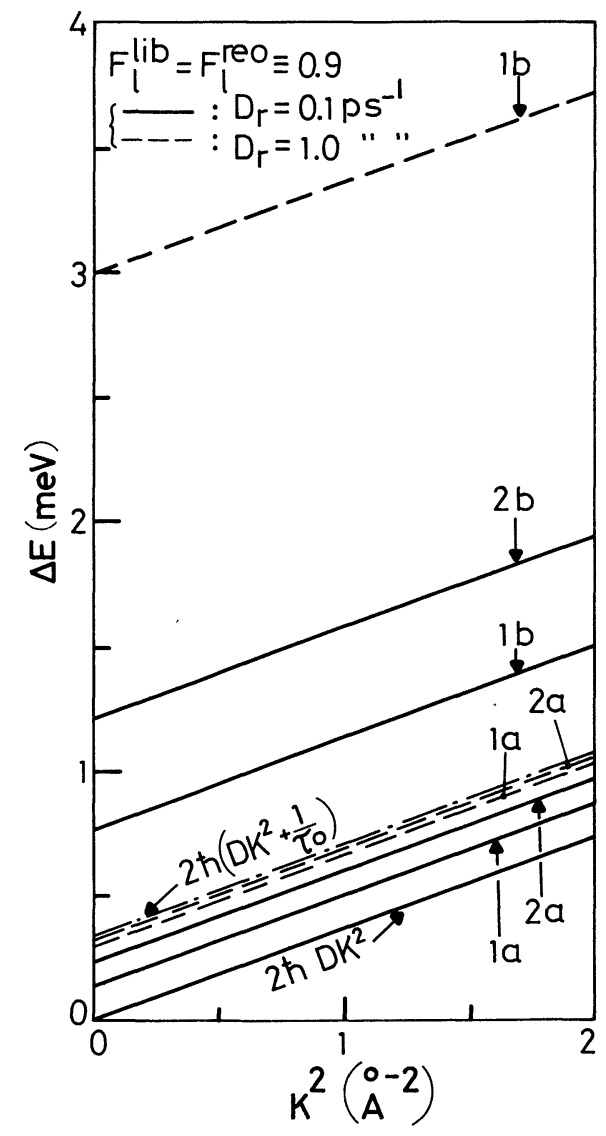

FIG. 1. - Calculated results of energy broadening $\Delta E$ in FWHM vs. squared momentum transfer $\kappa^{2}$ for the first few terms in the Lorentzian decomposition of the quasi-elastic scattering law. (The values for average lifetimes are taken as $\tau_{0}=\tau_{1}=4 \mathrm{ps}$.) of liquid benzene [5]. In liquid benzene, inelastic scattering due to librational or vibrational motions is observed in the region of energy transfer with $\hbar \omega \gtrsim 4 \mathrm{meV}$ [5], and so we should restrict the applicability of the present equation to the region below this value of the energy transfer.

The following values are used in the calculation, $D=2.75 \times 10^{-5} \mathrm{~cm}^{2} / \mathrm{s}, b=2.5 \AA, \tau_{0}=\tau_{1}=4 \mathrm{ps}$. The values for $\tau_{0}$ and $\tau_{1}$ used here are very rough estimates for those in liquid benzene. However, a small change in these values does not vary the structure of the results, but only shifts the energy broadenings by a constant and modifies the amplitudes a little.

We have no available experimental data for the values for $D_{\mathrm{r}}, F_{l}^{\text {lib }}$, and $F_{l}^{\text {reo }}$ in this liquid, and so trial calculations are made by using the following values for these parameters : $D_{\mathrm{r}}=0.1$ and $1.0 \mathrm{ps}^{-1}$, $F_{l}^{\text {lib }}=F_{l}^{\text {reo }}=0.9$ and $0.7(l \geqslant 1)$.

The results are shown in figures 1-3, where

$$
\begin{aligned}
A_{0} & =j_{0}^{2}(\kappa b), \\
A_{l} & =(2 l+1) j_{l}^{2}(\kappa b) \cdot \alpha_{l}, \\
B_{l} & =(2 l+1) j_{l}^{2}(\kappa b) \cdot \beta_{l}, \quad l \geqslant 1 .
\end{aligned}
$$

From figures 1 and 3, we notice that in the case of the smaller value for $D_{\mathrm{r}}$ and also the values for $F_{l}^{\text {lib }}$ and $F_{l}^{\text {reo }}$ near unity, one can obtain the energy broaden-

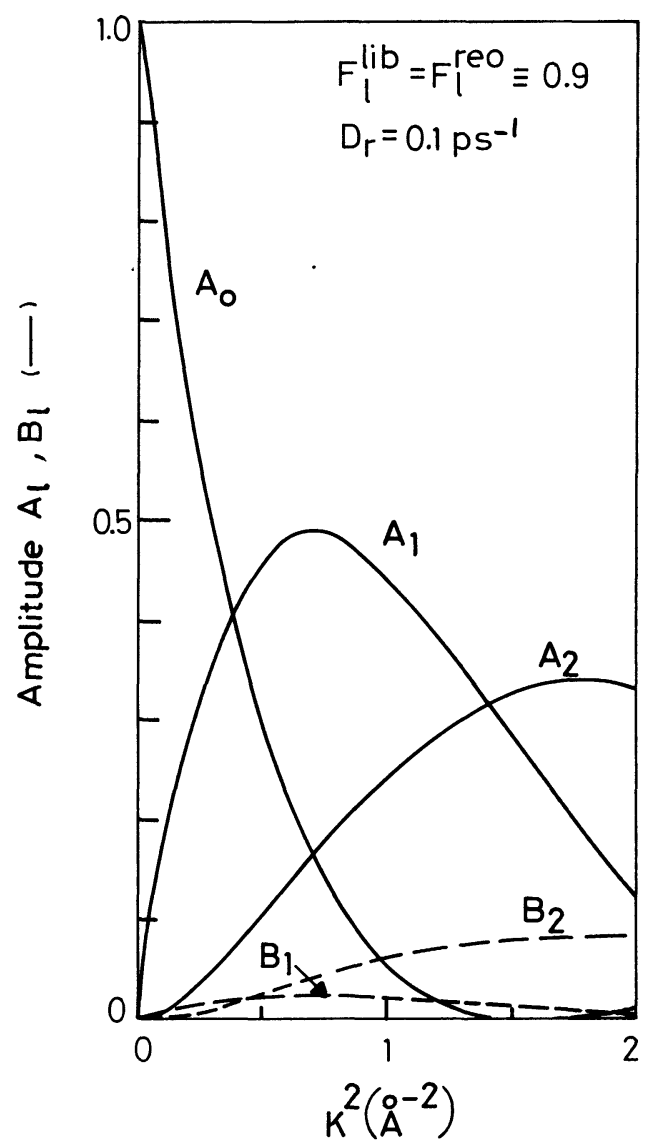

FIG. 2. - Calculated results for amplitudes of each of the Lorentzian terms in the quasi-elastic scattering law. (The values for $\tau_{0}$ and $\tau_{1}$ are the same as those in figure 1.) 
ing $\Delta E_{l a}$ for the term la well away from the asymptote of higher order terms $2 \hbar\left(D \kappa^{2}+\frac{1}{\tau_{0}}\right)$. Further, figure 2 shows that, between the two terms with equal $l$, the sharper one with the amplitude $A_{l}$ is always

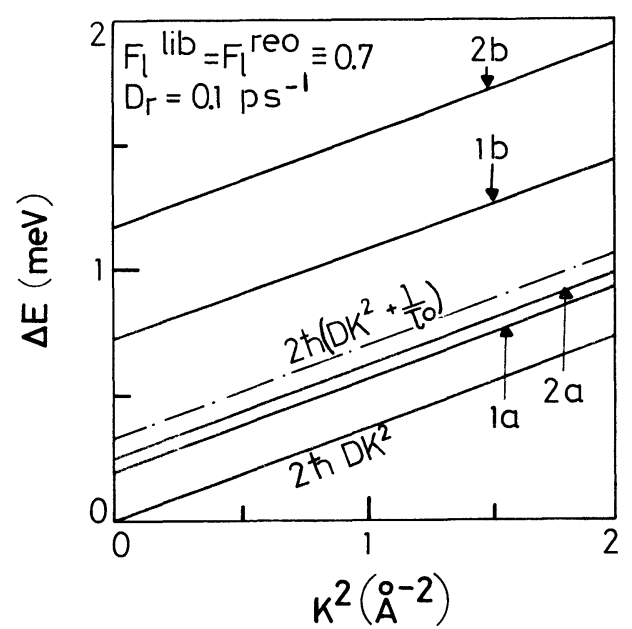

FIG. 3. - Calculated results of energy broadening by using smaller values for the expansion coefficients $F_{l}^{\text {lib }}$ and $F_{l}^{\text {reo }}$ of the orientational distribution functions. (The values for $\tau_{0}$ and $\tau_{1}$ are the same as those in figure 1.) predominant over the broader one with amplitude $B_{l}$. Figure 2 also shows that $A_{0}$ decreases rapidly when $\kappa^{2}$ increases, and instead another $A_{l}$ increases to become predominant in the larger $\kappa^{2}$ region.

5. Discussion. - The structures of energy broadening and amplitude mentioned above are much different from the reported results of the quasi-elastic scattering experiment on liquid benzene [5] where a single value of energy broadening was derived for each value of $\kappa^{2}$. Further, these values obtained are much larger than those expected from the first term in eq. (4), and approach those of higher order terms in the larger $\kappa^{2}$ region. Although our present result is based on certain assumptions on the molecular motions, it is very probable that, due to the effects of the experimental resolution, some mixture including different order terms were observed in the experiment over the whole region of $\kappa^{2}$. Separate observation of each of these terms will be possible in an appropriately selected region of $\kappa^{2}$ in recent experiments with higher energy resolution $[6,8]$.

Acknowledgments. - Much valuable discussions with Dr. R. E. Lechner during the author's stay at the Institut Laue-Langevin should be acknowledged concerning the present work.

\section{References}

[1] Sears, V. F., Can. J. Phys. 44 (1966) 1299; 45 (1966) 237. [2] SPRINGer, T., Springer Tracts in Modern Physics 64 (SpringerVerlag, Berlin) 1972.

[3] Larsson, K. E., J. Chem. Phys. 59 (1973) 4612

[4] Rosciszewski, K., Physica 75 (1974) 268.

[5] Trepădus, V.etal., J. Chem. Phys. 60 (1974) 2832.
[6] Volino, F. and Dianoux, A. J., Proc. EUCHEM Conf. Organic Liquids; Structures, Dynamics and Chemical Properties, Schloss Elmau (1976).

[7] Singwi, K. S. and SJölander, A., Phys. Rev. 119 (1960) 863. [8] Lechner, R. E., Proc. Conf. Neutron Scattering, Gatlinburg (1976) I, 310. 